\title{
Aqueous Phase Separation of Responsive Copolymers for Sustainable and Mechanically Stable Membranes
}

Wouter M. Nielen, Joshua D. Willott, and Wiebe M. de Vos*

Cite This: ACS Appl. Polym. Mater. 2020, 2, 1702-1710

Read Online

ABSTRACT: Membranes are often used in environmentally friendly applications and as a sustainable alternative to conventional processes. Unfortunately, the vast majority of polymeric membranes are produced via an unsustainable and environmentally unfriendly process that requires large amounts of harsh reprotoxic chemicals such as $\mathrm{N}$-methyl-2-pyrrolidinone and dimethylformamide. In this work, we investigate an aqueous phase separation (APS) system that uses weak polyelectrolytes, whose charge is dependent on the $\mathrm{pH}$ (weak polyelectrolytes), to produce membranes. Specifically the copolymer polystyrene-alt-maleic acid (PSaMA) is used. PSaMA contains responsive monomers, required for APS, and also unresponsive hydrophobic monomers that provide mechanical stability to the resultant membranes. This work demonstrates that by controlling the precipitation of PSaMA, it is possible to prepare a wide range of membranes; from microfiltration membranes capable of treating oily waste water to dense nanofiltration-type membranes with excellent micropollutant retentions and high mechanical stability. While similar materials in prior work could only withstand 4 bar, the membranes presented here demonstrate stable operation up to 20 bar. The only solvents used in this APS system are water and the green solvent acetic acid, thus making our APS process significantly more sustainable and environmentally friendly as compared to the conventional membrane fabrication methods.

KEYWORDS: polyelectrolytes, membranes, sustainable, water-based, aqueous phase separation

\section{INTRODUCTION}

Membrane technology is widely used for the production of drinking water, biomedical applications, waste water treatment, and chemical separations. ${ }^{1-3}$ Polymeric membranes, by far the most widely used membranes, are predominantly formed using the well-known nonsolvent-induced phase separation (NIPS) approach; a technique known for its versatility, low cost, and scalability. ${ }^{1-5}$ However, the solvents used in NIPS such as $N$ methyl-2-pyrrolidinone (NMP) and dimethylformamide (DMF) are expensive and reprotoxic, and over 50 billion liters of contaminated wastewater is produced annually. ${ }^{6}$ Recently, the use of NMP was restricted throughout the European Union by the Registration, Evaluation, Authorization, and Restriction of Chemicals legislation, and consequently the need to find suitable alternatives becomes even more pressing. ${ }^{7}$ Efforts to find alternatives to the conventional NIPS approach have been made. These include studies that aim to use less-toxic solvents such as dimethyl sulfoxide (DMSO), ionic liquids or super critical $\mathrm{CO}_{2}$, and other studies that use temperature-induced phase separation. ${ }^{8}$ However, all these approaches lack the versatility of NIPS, where extensive control over the membrane structure and pore size is the key to its success. More recently, de Vos proposed an aqueous phase separation (APS) approach similar to NIPS, which is based on the use of responsive weak polyelectrolytes, whose charge is dependent on the solution $\mathrm{pH}^{9}$ In this APS approach, water-soluble responsive polymers are dissolved in water and then precipitated also in water using a simple $\mathrm{pH}$ switch. The APS approach is schematically shown in Figure 1 for a weak polyacid. For APS, instead of a solvent exchange between NMP and water as shown in NIPS, a solvent exchange between alkaline and acidic water takes place. By using a polymer that is only soluble in either alkaline or acidic conditions, that is, a weak polyacid or a weak polybase, respectively, the system can undergo phase separation forming porous membranes.

In our previous work, we demonstrated the simplicity and versatility of the APS approach using the weak polybase poly(4-vinyl pyridine) (P4VP) by preparing both symmetric and asymmetric structures. ${ }^{10} \mathrm{P} 4 \mathrm{VP}$ is dissolved in water with a low $\mathrm{pH}$ where $\mathrm{P} 4 \mathrm{VP}$ is charged and, therefore, water soluble. A

Received: February 3, 2020

Accepted: March 6, 2020

Published: March 6, 2020 


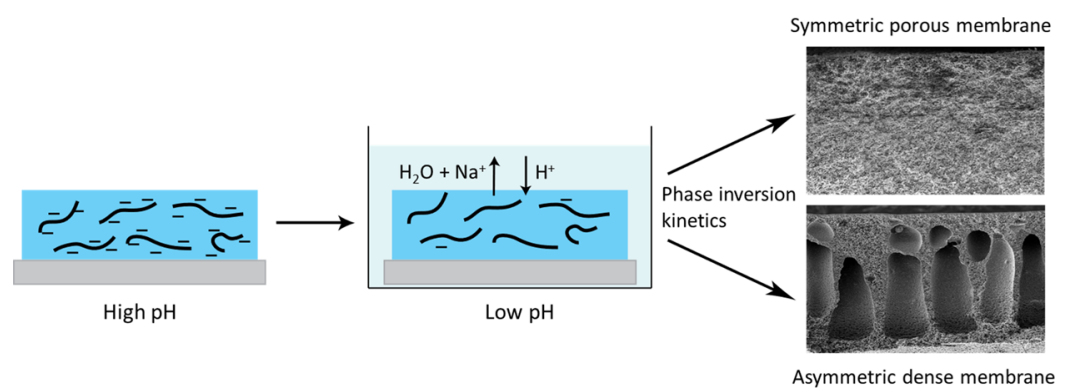

Figure 1. Schematic representation of APS using a weak polyacid.

thin film of the polymer solution is then placed in a coagulation bath with a high $\mathrm{pH}$ where P4VP loses its charge, becomes water insoluble, and then precipitates. By adjusting the polymer casting solution composition and the coagulation bath conditions, different membrane structures were obtained. A problem that was encountered was that the crosslinking steps required to improve the mechanical and chemical stability of the membranes resulted in quaternization of the amine groups, and thus, permanent charges were formed in the membrane leading to swelling. This swelling results in poor mechanical properties and unstable membrane performance above 4 bar of applied pressure. ${ }^{10}$

For APS to become a suitable sustainable alternative to NIPS, membranes with strong mechanical properties are essential. Ideally, one would use a responsive polymer which has high mechanical stability, that can be precipitated under mild conditions, and that does not require crosslinking or can be easily crosslinked under mild conditions. For this purpose, copolymers are expected to provide an ideal platform as a responsive monomer, required for APS, and can be combined with a monomer that exhibits good mechanical properties. Examples of such copolymers are the commercially available polystyrene-co-maleic acid (PScMA) partial isobutyl ester and copolymer polystyrene-alt-maleic acid (PSaMA). In these copolymers, the maleic acid monomer is the responsive unit that allows the polymer to dissolve in alkaline water and to precipitate in acidic water. Moreover, these carboxylic acid groups can be easily crosslinked in water under mild conditions. ${ }^{11}$ The styrene and isobutyl groups are expected to provide improved mechanical stability to the resultant membranes as they are unresponsive and hydrophobic and thus will reduce swelling.

The aim of this work is to demonstrate the potential of APS with copolymers as a sustainable alternative to conventional NIPS. For the first time, copolymers will be used in an APS approach. The behavior of the copolymers will be systemically evaluated using different acid concentrations, acid types, and additives in the polymer casting solution as well as the coagulation bath. By tuning these variables, the phase inversion kinetics will be controlled which in turn will allow us to prepare different types of membranes, from microfiltration type to nanofiltration type membranes, with improved mechanical properties.

\section{EXPERIMENTAL SECTION}

2.1. Materials. A PSaMA sodium salt solution $13 \%\left(M_{\mathrm{w}} 350,000\right)$, PScMA partial isobutyl ester $\left(M_{\mathrm{w}} 65,000 \mathrm{~g} \cdot \mathrm{mol}^{-1}\right)$, polyethylene glycol (PEG) $\left(M_{\mathrm{w}} 200 \mathrm{~g} \cdot \mathrm{mol}^{-1}\right.$, PEG 200; $M_{\mathrm{w}} 400 \mathrm{~g} \cdot \mathrm{mol}^{-1}$, PEG 400; $M_{\mathrm{w}} 600 \mathrm{~g} \cdot \mathrm{mol}^{-1}$, PEG 600; $M_{\mathrm{w}} 1500 \mathrm{~g} \cdot \mathrm{mol}^{-1}$, PEG 1500; $M_{\mathrm{w}} 2000 \mathrm{~g}$. $\mathrm{mol}^{-1}$, PEG 2000), and polyethyleneimine (PEI), branched $\left(M_{\mathrm{n}} 600\right.$ $\mathrm{g} \cdot \mathrm{mol}^{-1}$, PEI 600) were purchased from Sigma-Aldrich. N-(3-
Dimethylaminopropyl)- $N^{\prime}$-ethylcarbodiimide hydrochloride (EDC), N-hydroxysuccinimide (NHS), atenolol, atrazine, bezafibrate, bisphenol A, bromothymol blue, naproxen, phenolphthalein, sulphamethoxazole, oil red EGN, $n$-hexadecane, magnesium sulfate, magnesium chloride, sodium sulfate, sodium dodecyl sulfate (SDS), sodium hydroxide, glacial acetic acid, sodium phosphate monobasic dihydrate, phosphoric acid $85 \%$, and hydrochloric acid $37 \%$ were bought from Sigma-Aldrich. Ethanol of $100 \%$ technical grade was bought from Boom B.V. $n$-Hexane $99+\%$ was purchased from Acros Organics. Sodium chloride (Sanal P) was received from AkzoNobel. Deionized water (DI, $1.0 \mu \mathrm{S} \cdot \mathrm{cm}^{-1}$ ) was used for the preparation of coagulation baths and Milli-Q water (Millipore, $0.6 \mu \mathrm{S} \cdot \mathrm{cm}^{-1}$ ) was used to prepare solutions. The PSaMA solution was dried for $16 \mathrm{~h}$ at $100{ }^{\circ} \mathrm{C}$ to obtain the solid polymer which was used without further purification. All other chemicals were used as received.

2.2. Membrane Preparation. The polymer casting solutions were prepared by dissolving the polymer and additives in water, a typical solution consists of $18 \mathrm{~g}$ PSaMA $(20 \% \mathrm{w} / \mathrm{v}), 36 \mathrm{~mL}$ acetic acid (40\% v/v), and $36 \mathrm{~mL} \mathrm{H}_{2} \mathrm{O}(40 \% \mathrm{v} / \mathrm{v})$. The solutions were stirred until dissolved, followed by filtration through a Bekaert $25 \mu \mathrm{m}$ Bekipor ST25 AL 3 steel filter. The solutions were left for at least $24 \mathrm{~h}$ to allow air bubbles to leave the solution. The polymer solutions were cast onto a glass substrate using a steel casting knife with a $0.3 \mathrm{~mm}$ gap height. The films were then submerged in an acidic coagulation bath containing either $\mathrm{HCl}, \mathrm{H}_{3} \mathrm{PO}_{4}$, acetic acid, formic acid, malonic acid, $\mathrm{NaCl}$, or a combination thereof which induces the phase separation. After $5 \mathrm{~min}$, the formed porous film was transferred to a bath with $0.1 \mathrm{M} \mathrm{HCl}$ to wash salt and/or acids from the film. After a washing step of at least $30 \mathrm{~min}$, the membranes were washed twice more in $0.1 \mathrm{M} \mathrm{HCl}$. After the washing procedure, the membranes were crosslinked using EDC, NHS, and PEI. The crosslinker concentrations were based on the estimated amount of carboxylic acid groups per surface area of the membrane calculated using eq 1

$$
C=\frac{h \cdot A \cdot w \cdot a}{M_{\mathrm{w}}}
$$

where $C$ is the number of acid groups per surface area, $h$ is the casting height of the membrane, $A$ is the surface area of the membrane, $\mathrm{w}$ is the $\% \mathrm{w} / \mathrm{v}$ of the polymer in the casting solution, a is the amount of acid group per polymer chain, and $M_{\mathrm{w}}$ is the molecular weight of the polymer. A ratio of 1:1:0.4:0.33 of carboxylic acid groups/EDC/ NHS/PEI was used in the crosslinking reactions. The $\mathrm{pH}$ of the crosslinking mixture was set to approximately 5 using $\mathrm{HCl}$. After crosslinking, the membranes were washed twice for $30 \mathrm{~min}$ each in DI water and then stored in DI water.

2.3. Membrane Performance Tests. The performance of the membranes was studied using dead-end filtration cells with a pressurized vessel supplying the feed water. The free-standing membrane (a permeable surface area of $38 \mathrm{~mm}^{2}$ ) was supported by a polyphenylene sulfide nonwoven fabric and pure water permeability, and its ability to reject oil droplets, salts, PEG, or micropollutants was studied. Pure water permeability was measured by allowing the membrane to compact for $1 \mathrm{~h}$ after which the average permeability was recorded. Oil retention was measured using a stock oil-in-water emulsion, made by dissolving $0.2 \mathrm{~g}$ of oil red EGN in $6 \mathrm{~g}$ hexadecane; 
any undissolved solids were filtered off. ${ }^{12}$ Then, a $0.2 \% \mathrm{w} / \mathrm{w}$ oil-inwater emulsion was prepared by the addition of $0.463 \mathrm{~g} \cdot \mathrm{L}^{-1}$ SDS which was mixed for $15 \mathrm{~min}$ at $14,000 \mathrm{rpm}$. For oil filtration tests, the stock solution was diluted 20 times using $0.463 \mathrm{~g} \cdot \mathrm{L}^{-1} \mathrm{SDS}$. The diluted emulsion was stirred continuously during the measurement. Oil droplet retention was measured at $0.5 \mathrm{bar}$. The first $10 \mathrm{~min}$ of permeate was discarded after which samples were collected. Absorbance of the feed, retentate, and permeate was measured using UV/vis spectroscopy at $\lambda=523 \mathrm{~nm}$. The retention was calculated using the following equation

$$
R=\left(1-\frac{C_{\mathrm{p}}}{C_{\mathrm{f}}+C_{\mathrm{r}} / 2}\right) \times 100 \%
$$

where $R$ is the retention and $C_{\mathrm{p}}, C_{\mathrm{f}}$, and $C_{\mathrm{r}}$ are the absorbance of the permeate, feed, and retentate, respectively. Salt retention was measured at 4 bar of applied pressure using a $5 \mathrm{mM}$ salt solution $\left(\mathrm{MgSO}_{4}, \mathrm{MgCl}_{2}, \mathrm{Na}_{2} \mathrm{SO}_{4}\right.$, or $\left.\mathrm{NaCl}\right)$. The feed solution was stirred above the membrane and the conductivity of the feed, retentate, and permeate was measured. The retention was calculated using eq 2 , where $C_{\mathrm{p}}, C_{\mathrm{f}}$, and $C_{\mathrm{r}}$ are the conductivity in the permeate, feed, and retentate. The molecular weight cut-off (MWCO) of the membranes was measured using a $1 \mathrm{~g} \cdot \mathrm{L}^{-1}$ poly ethylene glycol solution with the following PEG molecular weights: 200, 400, 600, 1500, and $2000 \mathrm{~g}$. $\mathrm{mol}^{-1}$. Samples were analyzed via gel permeation chromatography (Agilent 1200/1260 Infinity GPC/SEC series, Polymer Standards Service data center and column compartment) using Milli- $Q$ eluent containing $50 \mathrm{mg} \cdot \mathrm{L}^{-1} \mathrm{NaN}_{3}$, at $1 \mathrm{~mL} \cdot \mathrm{min}^{-1}$, through two Polymer Standards Service Suprema $8 \times 300 \mathrm{~mm}$ columns in series: $1000 \AA$, 10 $\mu \mathrm{m}$ followed by $30 \AA, 10 \mu \mathrm{m}$. Concentrations were calculated via refractive index detection. Using eq 2, the retention for the different PEG molecules was calculated, where $R$ is the retention and $C_{\mathrm{p}}, C_{\mathrm{f}}$, and $C_{\mathrm{r}}$ are the concentrations in the permeate, feed, and retentate, respectively. Micropollutant retention was measured at 3 bar of applied pressure using a $3 \mathrm{mg} \cdot \mathrm{L}^{-1}$ solution of atenolol, atrazine, bezafibrate, bisphenol A, bromothymol blue, naproxen, phenolphthalein, and sulfamethoxazole. After two days of stirring, the solution $\mathrm{pH}$ was set to 5.8. To account for absorption of the micropollutants, the samples were taken after $24 \mathrm{~h}$ of continuous permeation at which point a steady state between absorption and desorption can be assumed. ${ }^{13}$ The samples were analyzed using ultra-high performance liquid chromatography (Dionex Ultimate 3000, water/acetonitrile gradient, $0.1 \%$ phosphoric acid, $0.8 \mathrm{~mL} \cdot \mathrm{min}^{-1}$ ) through a Thermo Scientific Acclaim RSLC 120 C18 column $(2.2 \mu \mathrm{m}, 2.1 \times 100 \mathrm{~mm})$, and micropollutant concentrations were determined via $\mathrm{UV} /$ vis detection at $225 \mathrm{~nm}$, bromothymol blue at $430 \mathrm{~nm}$. Micropollutant retention was determined using eq 2 .

2.4. Scanning Electron Microscopy. The crosslinked membranes were washed twice in ethanol and twice in $n$-hexane for $30 \mathrm{~min}$ to remove water from the membrane and to prevent pore collapse during drying. Noncrosslinked films were only washed in $n$-hexane as PSaMA dissolves in ethanol. After drying of the films, they were submerged in liquid nitrogen and fractured and then mounted on a sample holder. The samples were further dried in a vacuum oven at 30 ${ }^{\circ} \mathrm{C}$ for at least $4 \mathrm{~h}$, after which using a Quorum Q150T ES, $10 \mathrm{~nm}$ chromium was sputtered onto the samples. Images were taken with secondary electron imaging using JEOL JSM-6010LA and JSM-7610F scanning electron microscopes.

2.5. Nuclear Magnetic Resonance Imaging. PScMA (0.1 g) was dissolved in $0.5 \mathrm{~mL}$ deuterated $\mathrm{DMSO}$, and ${ }^{1} \mathrm{H}$ nuclear magnetic resonance (NMR) spectra were recorded using a Bruker $400 \mathrm{MHz}$ NMR spectrometer. Integrals of the intensity of characteristic peaks for each monomer were used to calculate the approximate average monomer distribution.

2.6. Dynamic Viscosity Measurements. The dynamic viscosity was measured using a HAAKE Viscotester 550 Rotational Viscometer with a SV-DIN rotor and cup, rotating at shear rates from 2.6 to 258 $\mathrm{s}^{-1}$ at $20^{\circ} \mathrm{C}$.

\section{RESULTS AND DISCUSSION}

3.1. Selection of Polyelectrolyte System. For APS with acidic polyelectrolytes, ideally a weak polyacid is used that precipitates under mild acidic conditions. As previously stated, copolymers are expected to provide a versatile platform for APS because a responsive monomer can be combined with a hydrophobic monomer. PScMA is one such polymer (see Figure $2 \mathrm{a}$ in the Experimental Section for the chemical
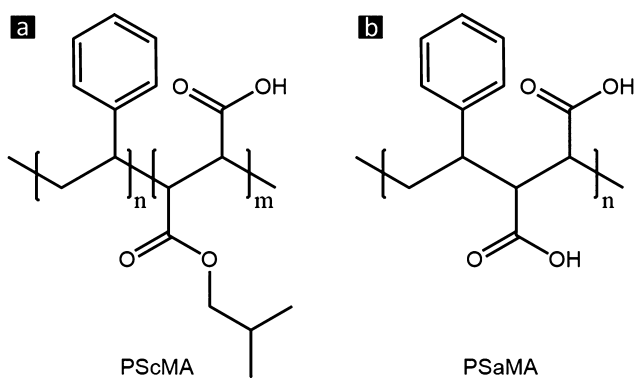

Figure 2. Chemical structures of (a) PScMA and (b) PSaMA.

structure of PScMA). In this random copolymer, the maleic acid monomers show the $\mathrm{pH}$-responsive behavior required for APS, while the styrene and partial isobutyl ester groups make the polymer more hydrophobic. This was hypothesized to result in precipitation at mild $\mathrm{pH}$ conditions. Using ${ }^{1} \mathrm{H}$ NMR, the monomer distribution was estimated to be approximately 1.5:1 mole ratio of styrene/maleic acid, with approximately half the acid groups having an isobutyl ester (see Figure S1 for further details). Dissolution of the polymer proved to be difficult under strong alkaline conditions of $\mathrm{pH}$ 14. Upon precipitation in the mildly acidic conditions of $\mathrm{pH} 3$, no stable polymer film was formed with the film fracturing into small pieces indicating inhomogeneous phase separation. This is not entirely surprising as the monomer distribution of PScMA is not homogeneous, and the isobutyl ester is partially hydrolyzed under strong alkaline conditions of the polymer casting solution. This inhomogeneous monomer distribution leads to the formation of hydrophilic and hydrophobic domains in the polymer chain. Supramolecular interactions between these domains most likely causes the film to fall apart. To overcome these issues, the alternating copolymer PSaMA was selected as a suitable alternative because it has a 1:1 ratio of hydrophilic and hydrophobic monomers, contains no hydrolyzable ester groups, and has a significantly higher molecular weight (see Figure $2 \mathrm{~b}$ in the Experimental Section for the chemical structure of PSaMA). As PSaMA has less hydrophobic groups, the polymer readily dissolves in water. Slightly more acidic conditions are required to induce precipitation of PSaMA when compared to PScMA. As expected, stable and dense films were formed by precipitating PSaMA solutions at $\mathrm{pH} 1$, as shown by the scanning electron microscopy (SEM) image presented in Figure 3a. The polymer concentration and the coagulation bath $\mathrm{pH}$ were varied systematically, but no porous structures were obtained. In conventional NIPS, it is not uncommon to add low molecular weight hydrophilic polymers such as short-chain PEG molecules into the polymer casting solution to improve the morphology. ${ }^{14-16}$ In this APS system, a similar effect is observed as porous films are formed when PEG is mixed into the polymer casting solution (see Figure $3 \mathrm{~b})$. However, the largely macrovoid structure is mechanically weak, and a large number of pinhole defects were observed in 


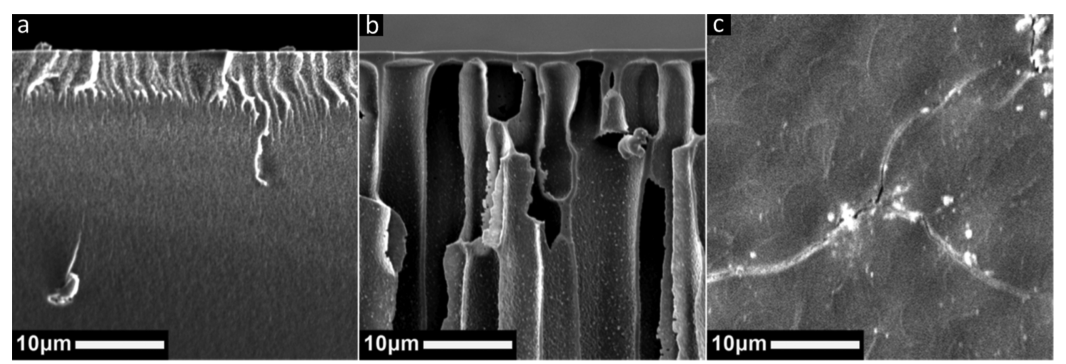

Figure 3. SEM images of (a) cross section of a film formed by precipitating $16.6 \% \mathrm{w} / \mathrm{v}$ PSaMA at $\mathrm{pH} 1$, a completely dense film is observed. (b,c) Cross section and the top layer of a film formed by precipitating $16.6 \% \mathrm{w} / \mathrm{v}$ PSaMA with $25 \% \mathrm{v} / \mathrm{v}$ PEG 200 at $\mathrm{pH} 0.5$, a thin dense top layer is observed which is supported by macrovoids. In the top layer, several defects can be observed which are most likely formed during drying of the film.

the top layer (Figure 3c), making these films unsuitable to be used for membrane applications.

Instead of adding short-chain PEG molecules, it is also possible to control precipitation kinetics by changing the polymer casting solution composition so that the solution is moved closer to the precipitation point. In conventional NIPS, this can be achieved by adding nonsolvent to the polymer solution which in this APS system means the addition of acid. For this purpose, weak acids were deemed to be more suitable than strong acids as they can be easily mixed into the solution while strong acids will induce local precipitation when added, making it more difficult to obtain homogenous polymer solutions. Acetic acid was chosen as it is a green solvent which can be produced in biorefineries and is easily recovered from waste water streams. ${ }^{17,18}$ Acetic acid is also an ideal acid for this system as its $\mathrm{p} K_{\mathrm{a}}$ value is 4.75 , which means that even at high concentrations acetic acid does not lower the solution $\mathrm{pH}$ below 2. Therefore, acetic acid brings the polymer solution close to the point of phase separation without actually causing precipitation of the polymer. However, when $20 \% \mathrm{v} / \mathrm{v}$ of acetic acid was added to a $20 \% \mathrm{w} / \mathrm{v}$ PSaMA solution, a gel-like phase was formed. It is interesting that when a higher concentration of acetic acid is used a translucent solution is formed, while further increases in acetic acid concentration result in a decrease in solution viscosity. Dynamic viscosity measurements reveal that a $20 \% \mathrm{w} / \mathrm{v}$ PSaMA solution with a $30 \% \mathrm{v} / \mathrm{v}$ acetic acid has a viscosity approximately 20 -fold higher than a $20 \%$ w/v PSaMA solution without acetic acid, as shown in Figure 4. When the acetic acid concentration is increased further to $40 \%$

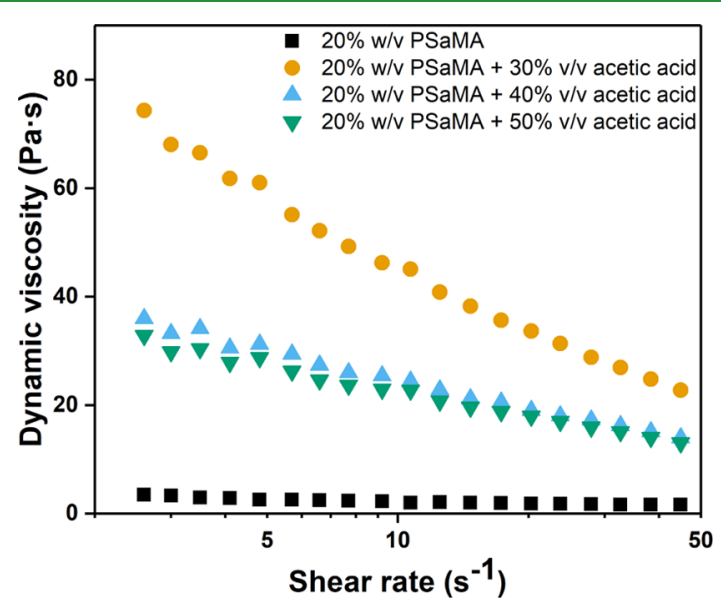

Figure 4. Dynamic viscosity for $20 \% \mathrm{w} / \mathrm{v}$ PSaMA solutions with different concentrations of acetic acid for different shear rates at 20 ${ }^{\circ} \mathrm{C}$. Data were taken from a single measurement. $\mathrm{v} / \mathrm{v}$, the solution viscosity is approximately halved. Further increasing the acetic acid to $50 \% \mathrm{v} / \mathrm{v}$ lowers the viscosity, but the effects are not as pronounced as the difference between the solutions with $30-40 \% \mathrm{v} / \mathrm{v}$ acetic acid.

The viscosity behavior presented in Figure 4 shows that acetic acid behaves as a nonsolvent at low concentrations and conversely as a solvent at higher concentrations for PSaMA. To explain this behavior, it is hypothesized that the decrease in solution $\mathrm{pH}$ by the addition of acetic acid causes partial protonation of the acid groups on the PSaMA. These protonated acid groups can then form interpolymer hydrogen bonding pairs which causes complexation/gelation of the polymer. Because acetic acid is a weak acid, it mostly exists in its associated form, and therefore, it can also form hydrogen bonding pairs with the protonated acid groups of the polymer. At higher concentrations, acetic acid disrupts the interpolymer hydrogen bonds through competitive binding which in turn prevents the formation of a polymer complex and thus results in a solution. Further increases in the acetic acid concentration decreases the interpolymer hydrogen bonding, and this results in the observed decrease in solution viscosity. Through its acidity, acetic acid is a nonsolvent for PSaMA, but at higher concentrations, acetic acid aids in the solvation of PSaMA through favorable hydrogen bonding interactions. However, it is expected that at even higher acetic acid concentrations, the amount of water in the solution will be too low to dissolve PSaMA. Together this means that the acetic acid concentration is an additional parameter to control the phase inversion kinetics. In conventional NIPS, an approach to increase the porosity of the membrane is to add solvent to the coagulation bath to delay polymer demixing. ${ }^{3}$ For this APS system, the same effect can be achieved by adding acetic acid to the coagulation bath, which will be discussed in the following section.

3.2. Formation of Porous Membranes. When the $20 \%$ $\mathrm{w} / \mathrm{v}$ PSaMA solution with $40 \% \mathrm{v} / \mathrm{v}$ acetic acid is submerged in the coagulation bath, the change in environmental $\mathrm{pH}$ causes protonation of the acid groups. This results in a decrease of the hydrophilicity of PSaMA as it loses its charge, while at the same time the hydrogen bonding capacity is increased. This makes it thermodynamically unfavorable for PSaMA to remain in solution, and thus, it precipitates. By the addition of acetic acid in the coagulation bath, interpolymer hydrogen bonding is suppressed, and therefore, acetic acid can be used to tune the solvent quality for both the polymer solution and the coagulation bath. Upon addition of $2 \mathrm{M}$ acetic acid to the coagulation bath, a slow and delayed demixing is observed (see the Supporting Information for a short movie of the phase separation process), which results in the formation of 

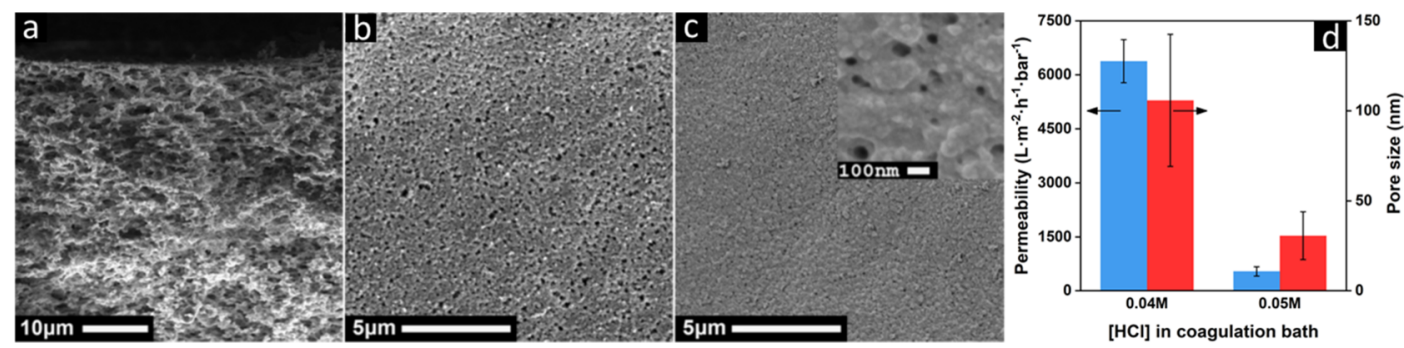

Figure 5. (a,b) SEM images of the cross section and top layer of a membrane formed by precipitating a $20 \% \mathrm{w} / \mathrm{v}$ PSaMA solution with $40 \% \mathrm{v} / \mathrm{v}$ acetic acid, in a coagulation bath with $2 \mathrm{M}$ acetic acid, $0.1 \mathrm{M} \mathrm{NaCl}$, and $0.04 \mathrm{M} \mathrm{HCl}$. (c) SEM images of the top layer of a membrane formed by precipitating a $20 \% \mathrm{w} / \mathrm{v}$ PSaMA solution with $40 \% \mathrm{v} / \mathrm{v}$ acetic acid, in a coagulation bath with $2 \mathrm{M}$ acetic acid, $0.1 \mathrm{M} \mathrm{NaCl}$, and $0.05 \mathrm{M} \mathrm{HCl}$. (d) Pure water permeability and average pore size of the membranes, as shown in $(a-c)$. Permeability was measured in a dead-end filtration setup at 1 bar, the data shown are the average of at least three different membranes with the error bars showing the sample standard deviation. The average pore size is determined using ImageJ software, the error bars display the population standard deviation (see Figure S2 for pore size distribution).

symmetric porous films (see Figure $5 a-c$ ). From SEM topsection image analysis, the pore size is on an average $106 \pm 37$ $\mathrm{nm}$, indicating that it is a microfiltration membrane (see Figure S2a) for pore size distribution data. In addition to acetic acid, $0.1 \mathrm{M} \mathrm{NaCl}$ was also used in the coagulation bath as it helped to provide stable films. It is expected that the salt can affect the phase separation as it is known that the salt concentration can have a strong effect on polyelectrolyte conformation. ${ }^{19}$ However, this was not further investigated. To ensure that PSaMA does not redissolve during the membrane performance tests, the membranes were crosslinked in water using a small amount of low molecular weight branched PEI, EDC, and NHS, which links the amine groups of PEI to the carboxylic acid groups of PSaMA. ${ }^{11}$ Branched PEI was chosen as the crosslinker because it has multiple primary amine groups, and it is also much safer to use than smaller molecules with multiple primary amine groups. After crosslinking, not only the chemical stability but also the mechanical stability of the porous membranes was visibly improved. Pure water permeability measurements show a permeability of approximately $6500 \mathrm{~L} \cdot \mathrm{m}^{-2} \cdot \mathrm{h}^{-1} \cdot \mathrm{bar}^{-1}$, which is in line with commercial microfiltration membranes. ${ }^{1,2}$ To study membrane retention, an oil-in-water emulsion stabilized by SDS was prepared with an average droplet size of 3-5 $\mu \mathrm{m} .{ }^{12}$ Because of the dead-end filtration setup, the membrane rapidly fouls resulting in a significant drop in water permeability. Therefore, retention was measured during the first $15 \mathrm{~min}$ of permeation to measure the actual performance of the membrane and not that of the fouling layer that forms. It is expected that the retention will remain stable or even improve over time as an additional oil "cake" layer is formed on top of the membrane. The membranes were found to retain over $98 \%$ of oil demonstrating their function as a microfiltration membrane.

Further investigations on the effect of acetic acid concentration in the coagulation bath revealed that the obtained membrane structures were similar at the concentrations of 2.5 and $3 \mathrm{M}$ acetic acid. However, when an acetic acid concentration of $1.5 \mathrm{M}$ or lower was used in the coagulation bath, the films were mechanically unstable and could not be removed from the casting substrate intact, indicating a much weaker structure. As the change in $\mathrm{pH}$ is the main driving force to precipitate PSaMA, the $\mathrm{pH}$ of the coagulation bath was systemically investigated. With only a slight increase in the $\mathrm{HCl}$ concentration from 0.04 to $0.05 \mathrm{M}$, a significant reduction in the membrane pore size and pure water permeability was observed (Figure 5c,d). Analysis of the SEM images show that the average pore size is $31 \pm 13 \mathrm{~nm}$, indicating that it is an open ultrafiltration membrane (see Figure S2b for pore size distribution data). Further increases in the $\mathrm{HCl}$ concentration in the coagulation bath resulted in the formation of a dense top layer with macrovoids and pinhole defects. When a $\mathrm{HCl}$ concentration of $0.03 \mathrm{M}$ or lower was used in the coagulation bath, highly porous symmetric structures were formed with high permeabilities; however, these membranes lacked mechanical stability. Here, a greater than $50 \%$ drop in permeability was observed within $1 \mathrm{~h}$ of filtration indicating significant structural collapse. This shows the sensitivity as well as the versatility of the APS process. By controlling the acid concentration, different open membrane structures can be prepared in a simple way without the use of harsh chemicals.

3.3. Formation of Membranes with Dense Top Layers. Acetic acid was chosen for the coagulation bath as it is also used in the polymer casting solution, but there are other weak acids that could be used. To investigate the effect of the type of acid used in the coagulation bath on membrane formation, several other weak acids with different $\mathrm{p} K_{\mathrm{a}}$ values than acetic acid are investigated, as shown in Table 1.

Table 1. Chemical Structure and $\mathrm{p} K_{\mathrm{a}}$ Values of the Selected Acids $^{20}$

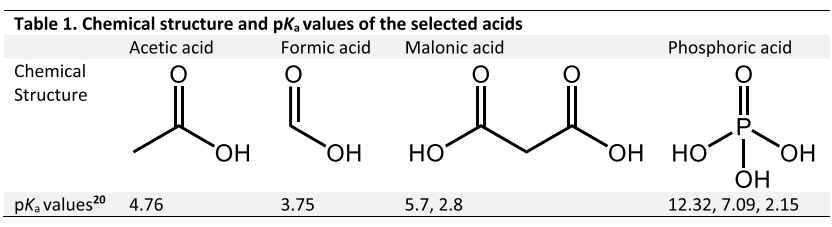

Formic acid, malonic acid, and phosphoric acid were chosen as these acids can all form hydrogen bonding pairs with protonated carboxylic acid groups in a similar fashion as acetic acid. Formic acid has a similar structure as acetic acid, while malonic acid and phosphoric acid are diprotic and triprotic acids, respectively, and they can potentially form hydrogen bonding pairs with multiple protonated carboxylic acid groups. Moreover, because of the lower $\mathrm{p} K_{\mathrm{a}}$ values of these acids, additional $\mathrm{HCl}$ in the coagulation bath was not needed in order to precipitate PSaMA. For all acids, a $2 \mathrm{M}$ concentration was used which resulted in asymmetric structures with dense top layers and macrovoid defects in the support structure (Figure $6 a-f)$. The high viscosity of the solution, as shown in Figure 4, was expected to suppress macrovoid formation, yet the support structure is dominated by them. It is therefore 


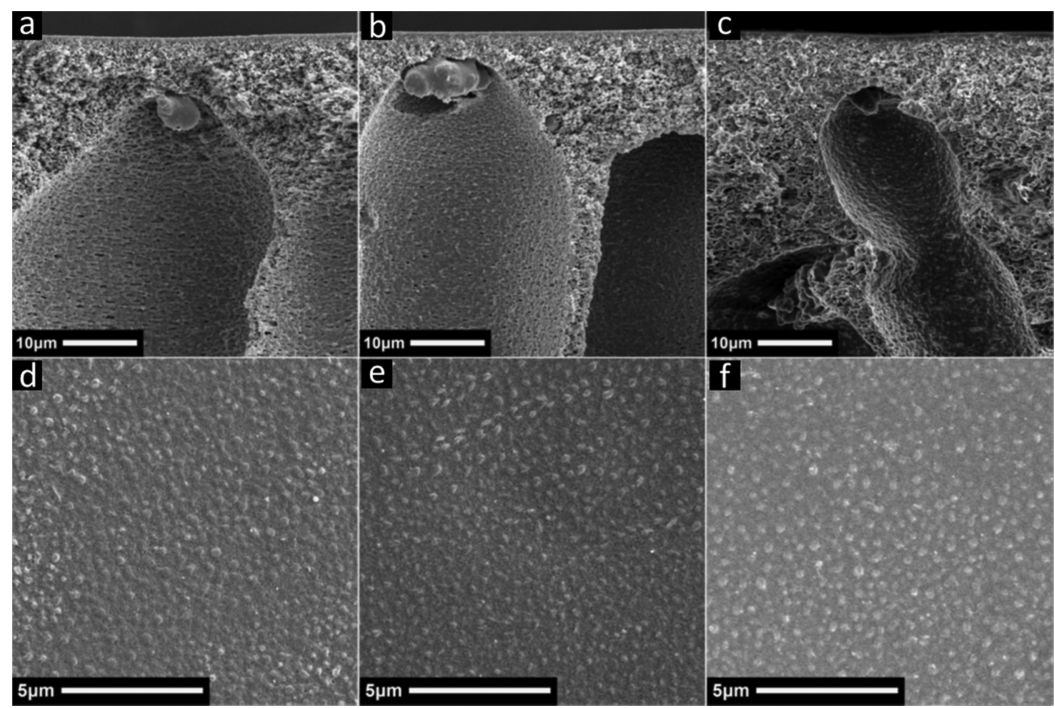

Figure 6. SEM images of the cross section and the corresponding top surfaces of membranes formed with $2 \mathrm{M}$ formic (a,d), $2 \mathrm{M}$ malonic (b,e), or $2 \mathrm{M}$ phosphoric acid (c,f) in the coagulation bath using $20 \% \mathrm{w} / \mathrm{v}$ PSaMA with $40 \% \mathrm{v} / \mathrm{v}$ acetic acid as the casting solution. The structures appear to be similar regardless of the acid that is used in the coagulation bath.

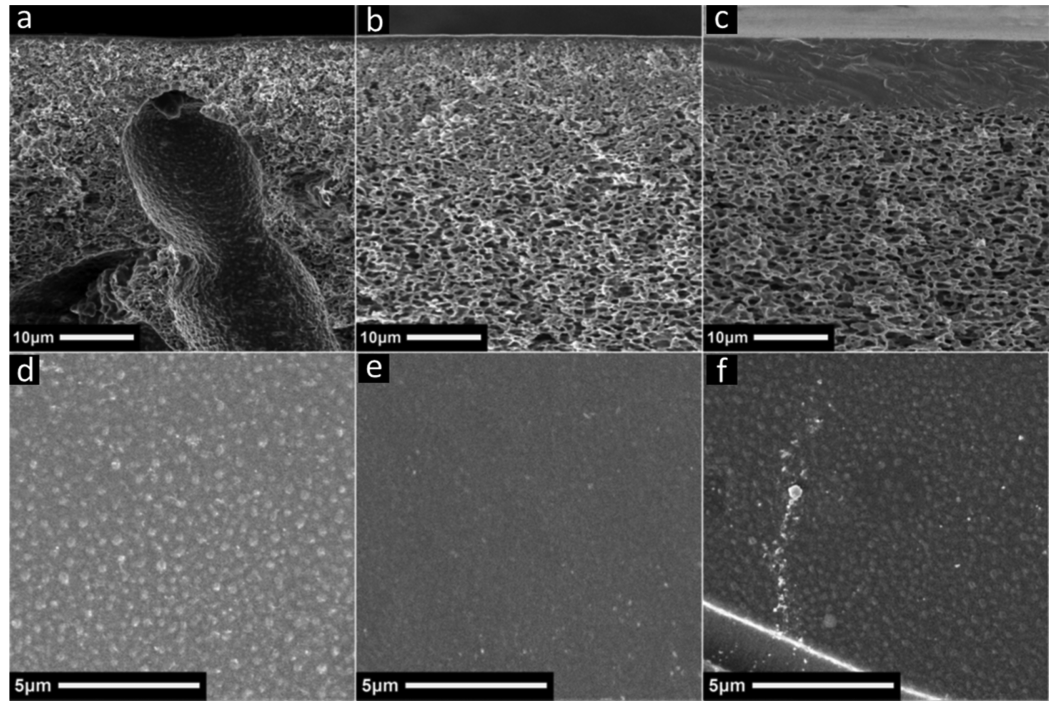

Figure 7. SEM images of the cross section and the corresponding top surfaces of membranes formed with $2 \mathrm{M}$ (a,d), $2.5 \mathrm{M}$ (b,e), or $3 \mathrm{M}$ phosphoric acid (c,f) in the coagulation bath using $20 \% \mathrm{w} / \mathrm{v}$ PSaMA with $40 \% \mathrm{v} / \mathrm{v}$ acetic acid as the casting solution. Increasing the phosphoric acid concentration from 2 to $2.5 \mathrm{M}$ results in a suppression of macrovoids. Further increasing the concentration to $3 \mathrm{M}$ increases the thickness of top layer from $007 \mathrm{E} 300 \mathrm{~nm}$ to hydrolyzable $10 \mu \mathrm{m}$, which cracks upon drying.

expected that a dense selective layer is formed, which by impeding the diffusion of acid slows down the phase inversion, causing the formation of macrovoids. This is confirmed by the observation that for all three acids, the initial onset of the phase separation is near instant instead of the delayed onset that was observed when acetic acid was used in the coagulation bath, yet it takes quite a while before separation is complete (see the Supporting Information for a short movie of the phaseseparation process).

The membranes prepared with phosphoric acid in the coagulation bath were studied in more detail by changing the coagulation bath acid concentration. It was observed that at all phosphoric acid concentrations, a dense top layer without visible pores is formed (Figure $7 \mathrm{~d}-\mathrm{f}$ ) and when a concentration lower than $2 \mathrm{M}$ is used, the overall structures are similar (Figures 7a and S3). However, when a phosphoric acid concentration higher than $2 \mathrm{M}$ is used in the coagulation bath, significant changes in the support structure are observed. At $2.5 \mathrm{M}$, as shown in Figure $7 \mathrm{~b}$, the macrovoid defects are almost completely suppressed, and at $3 \mathrm{M}$, the thickness of the top layer increases from approximately $300 \mathrm{~nm}$ to $007 \mathrm{E} 10 \mu \mathrm{m}$ (Figure 7c). A possible explanation for this behavior is that at $2.5 \mathrm{M}$, the phosphoric acid concentration is high enough to diffuse through the dense selective layer fast enough so that in combination with viscosity of the solution (Figure 4), marcovoids are suppressed. At $3 \mathrm{M}$ concentration, the ability of phosphoric acid to form hydrogen bonding pairs with two different carboxylic acid groups could possibly cause severe densification of the membrane structure which therefore creates a very thick dense layer. Further research is required to confirm this and to determine whether this effect also applies to other weak acids such as malonic acid that can also 

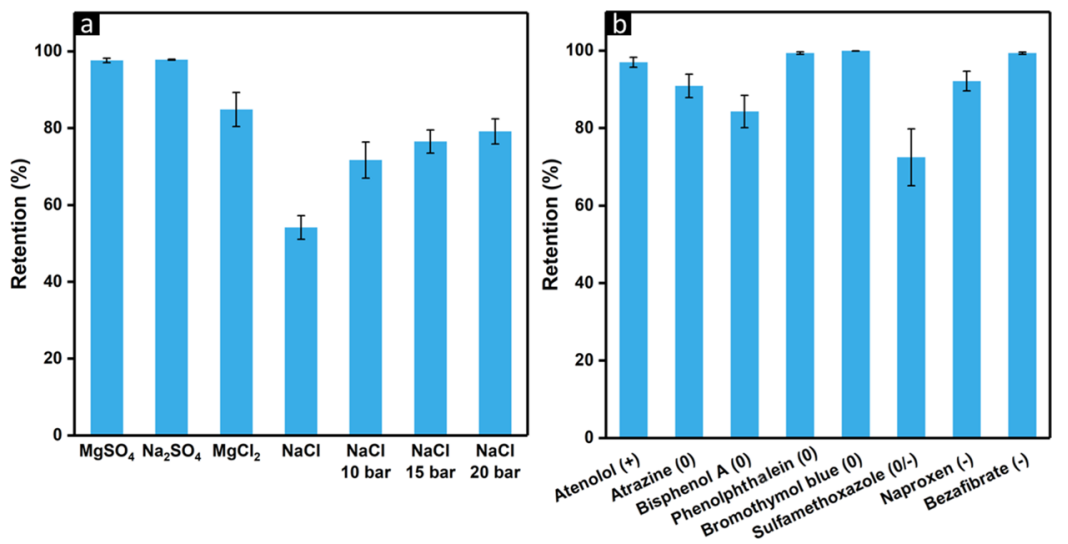

Figure 8. Retention of salt (a) and micropollutants (b) by membranes formed with $2 \mathrm{M}$ phosphoric acid in the coagulation bath using $20 \% \mathrm{w} / \mathrm{v}$ PSaMA with $40 \% \mathrm{v} / \mathrm{v}$ acetic acid as the casting solution. All measurements were performed using dead-end filtration cells using either $4,10,15$, or 20 bars of pressure for the salt retention and 3 bars of pressure for the micropollutant retention. The $\mathrm{pH}$ of the micropollutant mix was set to 5.8; in this regime, the micropollutants either have a positive $(+)$, negative $(-)$, or no charge $(0)$, with sulfamethoxazole being partially charged. Data shown are the average of at least three different membranes with the error bars showing the sample standard deviation.

from hydrogen bonding pairs with multiple carboxylic acid groups. For the membranes prepared with $0.5-2.5 \mathrm{M}$ phosphoric acid in the coagulation bath, pure water permeability and $\mathrm{MgSO}_{4}$ retentions were measured. All membranes have similar performance with high $\mathrm{MgSO}_{4}$ retentions (>94\%) and relatively low water permeability $\left(0.35 \pm 0.1 \mathrm{~L} \cdot \mathrm{m}^{-2} \cdot \mathrm{h}^{-1} \cdot \mathrm{bar}^{-1}\right)$. Based on the $\mathrm{MgSO}_{4}$ retention data, the membranes can be compared to nanofiltration membranes prepared by classical processes such as layer-bylayer deposition of polyelectrolytes or interfacial polymerization on top of porous NIPS-based polymeric membranes. $^{21,22}$ The advantage of this APS system compared to layer-by-layer deposition or interfacial polymerization is that the APS membranes are prepared in a single step followed by submersion in a crosslinking solution. This approach can be easily configured to a roll-to-roll process that will most likely result in a significant time reduction to produce these APS membranes. Although the water permeability is currently low, APS is still in its infancy and there remain plenty of opportunities for optimization, for example, in the polymer solution, the coagulation bath, or in the selection of polymer. The simplicity in preparation and the possibility to prepare open porous microfiltration and ultrafiltration membranes as well as nanofiltration membranes with dense top layers by just changing the type of acid, and the acid concentration shows again that APS is a promising sustainable alternative to the traditional NIPS process.

3.4. Detailed Dense Membrane Separation Performance. The membranes prepared with $2 \mathrm{M}$ phosphoric acid in the coagulation bath were studied in greater detail. As shown in Figure 8a, retentions for different monovalent and divalent salts are compared at 4 bar of applied pressure. In addition, the retention of $\mathrm{NaCl}$ was measured at 10, 15, and 20 bar. As expected, the retention is lowest for $\mathrm{NaCl}$ which can be attributed to its small size and monovalency. The higher retention for $\mathrm{MgCl}_{2}$ compared to $\mathrm{NaCl}$ is also expected as $\mathrm{Mg}^{2+}$ is divalent and has a larger hydrated diameter than $\mathrm{Na}^{+}$. The difference between $\mathrm{MgCl}_{2}$ and $\mathrm{Na}_{2} \mathrm{SO}_{4}$ can be attributed to Donnan exclusion as the negative charge of PSaMA gives the membrane an overall net negative charge. ${ }^{23}$ At higher pressures, namely 10,15 , and 20 bar, the $\mathrm{NaCl}$ retention increases and permeability remains constant indicating that the solution-diffusion mechanism applies, and the separation is not based on pore size exclusion. ${ }^{1,2}$ The membranes show excellent stability under high transmembrane pressures as the water permeability remained stable at $0.32 \pm 0.04 \mathrm{~L} \cdot \mathrm{m}^{-2} \cdot \mathrm{h}^{-1} \cdot \mathrm{bar}^{-1}$ over the course of five days under pressures ranging from 10 to 20 bar, showing that no membrane densification occurs. In addition, after the membrane performance at 20 bar transmembrane pressure was measured, it was measured again at 10 bar transmembrane pressure which showed the same performance as before. MWCO measurements were performed to assess the capability of this membrane to retain small organic molecules. The retention of differently sized uncharged PEG molecules was analyzed, and PEG molecules with a molecular weight of $232 \pm 27 \mathrm{Da}$ or larger were retained ( $\geq 90 \%)$, as shown in Figure S4. MWCO measurements using PEG molecules is a model system, and therefore, additional experiments were performed where the retention of a mixture of charged and uncharged small organic molecules was studied (see Figure $8 \mathrm{~b}$ ). These so-called micropollutant molecules were chosen for their range of low molecular weights (215$624 \mathrm{~g} \cdot \mathrm{mol}^{-1}$ ) and different charges which provides a better representation of the membrane performance under more realistic conditions. ${ }^{13}$ An average retention of $92 \%$ shows that these membranes are capable of removing a diverse range of micropollutants from water, and this performance is on par or even better when compared to what is obtained with conventional membranes in the literature. ${ }^{24}$ The retention for the largest micropollutant, bromothymol blue, is more than $99.9 \%$, which importantly shows that the top separation layer of this membrane is defect free. Differences in retention of the different micropollutants can be attributed to their differences in charge, size, geometry, and affinity to the membrane material. $^{24}$

\section{CONCLUSIONS}

A simple APS approach based on a $\mathrm{pH}$ switch with responsive copolymers has been used to prepare a wide range of mechanically stable membranes without using unsustainable and toxic organic solvents such as NMP and DMF. Similar to the classical NIPS process, the phase inversion kinetics can be controlled by changing the composition of the polymer casting solution and the coagulation bath. By controlling the $\mathrm{pH}$ as 
well as the type and concentration of acid used in the casting solution and coagulation bath, microfiltration, ultrafiltration, and nanofiltration membranes with dense top layers have been successfully prepared. This clearly showcases the simplicity and versatility of the APS approach. The open porous membranes can be used to effectively treat oily water streams, $>98 \%$ rejection, while the membranes with thin dense separation layers show excellent retentions toward small, notoriously difficult to remove micropollutants with an average rejection (> 92\%). In addition to possessing excellent retention performance, the nanofiltration type membranes have significantly improved mechanical stability, in comparison to similar materials in our earlier work, ${ }^{10}$ with consistent performance up to 20 bar of applied pressure. Here, the carboxylic acid groups within the PSaMA copolymer are easily crosslinked under mild conditions in water, which provides greater mechanical stability and also chemical stability for the membranes in nonacidic media while maintaining an overall green and sustainable fabrication process. This research shows that APS can be used to prepare different types of highly performing membranes and therefore has the potential to become a viable alternative to the classical NIPS process.

\section{ASSOCIATED CONTENT}

\section{SI Supporting Information}

The Supporting Information is available free of charge at https://pubs.acs.org/doi/10.1021/acsapm.0c00119.

${ }^{1} \mathrm{H}$ NMR spectra of PScMA; pore size distribution data for membranes, as shown in Figure 4; SEM images of membranes prepared in $\mathrm{H}_{3} \mathrm{PO}_{4}$ concentrations from 0.5 to $1.5 \mathrm{M}$; MWCO data for membranes discussed in Section 3.4; and movies of the phase inversion process of membranes discussed in Sections 3.2 and 3.3 (PDF).

\section{AUTHOR INFORMATION}

\section{Corresponding Author}

Wiebe M. de Vos - Membrane Surface Science (MSuS), Membrane Science and Technology Cluster, Mesa+ Institute for Nanotechnology, University of Twente 7500 AE Enschede, the Netherlands; (1) orcid.org/0000-0002-0133-1931; Phone: +31534894495; Email: w.m.devos@utwente.nl

\section{Authors}

Wouter M. Nielen - Membrane Surface Science (MSuS), Membrane Science and Technology Cluster, Mesa+ Institute for Nanotechnology, University of Twente 7500 AE Enschede, the Netherlands

Joshua D. Willott - Membrane Surface Science (MSuS), Membrane Science and Technology Cluster, Mesa+ Institute for Nanotechnology, University of Twente 7500 AE Enschede, the Netherlands; (1) orcid.org/0000-0003-1870-755X

Complete contact information is available at:

https://pubs.acs.org/10.1021/acsapm.0c00119

\section{Notes}

The authors declare the following competing financial interest(s): The university of Twente has filed a patent application related to the research in this work (PCT/ NL2018/050742). The authors on this manuscript are listed as the inventers of this patent.

\section{ACKNOWLEDGMENTS}

The authors acknowledge funding support from the "Vemieuwingsimpuls" program through project VIDI 723.015.003 (financed by the Netherlands Organization for Scientific Research, NWO) and from the European Research Council (ERC) under the European Union's Horizon 2020 research and innovation program (ERC StG 714744 SAMBA).

\section{REFERENCES}

(1) Baker, R. W. Membrane Technology and Applications; John Wiley \& Sons, 2012.

(2) Strathmann, H.; Giorno, L.; Drioli, E. Introduction to Membrane Science and Technology; Wiley-VCH Weinheim, 2011.

(3) Mulder, J. Basic Principles of Membrane Technology; Springer Science \& Business Media, 2012.

(4) Loeb, S.; Sourirajan, S. Sea water demineralization by means of an osmotic membrane. Advances in Chemistry; University of California, 1962, Vol. 38, pp 117-132.

(5) Guillen, G. R.; Pan, Y.; Li, M.; Hoek, E. M. V. Preparation and Characterization of Membranes Formed by Nonsolvent Induced Phase Separation: A Review. Ind. Eng. Chem. Res. 2011, 50, 37983817.

(6) Razali, M.; Kim, J. F.; Attfield, M.; Budd, P. M.; Drioli, E.; Lee, Y. M.; Szekely, G. Sustainable wastewater treatment and recycling in membrane manufacturing. Green Chem. 2015, 17, 5196-5205.

(7) European Chemical Agency. https://echa.europa.eu/substanceinformation/-/substanceinfo/100.011.662 (accessed Feb 3, 2020).

(8) Figoli, A.; Marino, T.; Simone, S.; Di Nicolò, E.; Li, X.-M.; He, T.; Tornaghi, S.; Drioli, E. Towards non-toxic solvents for membrane preparation: a review. Green Chem. 2014, 16, 4034-4059.

(9) de Vos, W. M. (Universiteit Twente), Aqueous phase separation method. U.S. Patent 20,180,318,775 A1, Application no. 15/972,273, 2018.

(10) Willott, J. D.; Nielen, W. M.; de Vos, W. M. Stimuli-Responsive Membranes through Sustainable Aqueous Phase Separation. ACS Appl. Polym. Mater. 2020, 2, 659-667.

(11) Sehgal, D.; Vijay, I. K. A method for the high efficiency of water-soluble carbodiimide-mediated amidation. Anal. Biochem. 1994, 218, 87-91.

(12) Dickhout, J. M.; Kleijn, J. M.; Lammertink, R. G. H.; De Vos, W. M. Adhesion of emulsified oil droplets to hydrophilic and hydrophobic surfaces - effect of surfactant charge, surfactant concentration and ionic strength. Soft Matter 2018, 14, 5452-5460.

(13) Ilyas, S.; Abtahi, S. M.; Akkilic, N.; Roesink, H. D. W.; de Vos, W. M. Weak polyelectrolyte multilayers as tunable separation layers for micro-pollutant removal by hollow fiber nanofiltration membranes. J. Membr. Sci. 2017, 537, 220-228.

(14) Yang, G.; Zhang, L.; Feng, H. Role of polyethylene glycol in formation and structure of regenerated cellulose microporous membrane. J. Membr. Sci. 1999, 161, 31-40.

(15) Ma, Y.; Shi, F.; Ma, J.; Wu, M.; Zhang, J.; Gao, C. Effect of PEG additive on the morphology and performance of polysulfone ultrafiltration membranes. Desalination 2011, 272, 51-58.

(16) Boom, R. M.; Wienk, I. M.; Van den Boomgaard, T.; Smolders, C. A. Microstructures in phase inversion membranes. Part 2. The role of a polymeric additive. J. Membr. Sci. 1992, 73, 277-292.

(17) Prat, D.; Hayler, J.; Wells, A. A survey of solvent selection guides. Green Chem. 2014, 16, 4546-4551.

(18) Pal, P.; Nayak, J. Acetic acid production and purification: critical review towards process intensification. Sep. Purif. Rev. 2017, 46, 44-61.

(19) Barrat, J.-L.; Joanny, J.-F. Theory of polyelectrolyte solutions. Adv. Chem. Phys. 1996, 94, 1-66.

(20) PubChem. https://pubchem.ncbi.nlm.nih.gov/ (accessed Feb $3,2020)$.

(21) de Grooth, J.; Oborný, R.; Potreck, J.; Nijmeijer, K.; de Vos, W. $M$. The role of ionic strength and odd-even effects on the properties 
of polyelectrolyte multilayer nanofiltration membranes. J. Membr. Sci. 2015, 475, 311-319.

(22) Zhang, Z.; Kang, G.; Yu, H.; Jin, Y.; Cao, Y. From reverse osmosis to nanofiltration: Precise control of the pore size and charge of polyamide membranes via interfacial polymerization. Desalination 2019, 466, 16-23.

(23) Schaep, J.; Van der Bruggen, B.; Vandecasteele, C.; Wilms, D. Influence of ion size and charge in nanofiltration. Sep. Purif. Technol. 1998, 14, 155-162.

(24) te Brinke, E.; Reurink, D. M.; Achterhuis, I.; de Grooth, J.; de Vos, W. M. Asymmetric polyelectrolyte multilayer membranes with ultrathin separation layers for highly efficient micropollutant removal. Appl. Mater. Today 2020, 18, 100471. 